\title{
The effect of dietary lipid sources on layer fertility and hatchability
}

\author{
O.S. Olubowale ${ }^{1}$, F.H. de Witt ${ }^{1 \#}$, J.P.C. Greyling ${ }^{1}$, A. Hugo ${ }^{2}$, A.M. Jooste ${ }^{1}$ \\ \& M.B. Raito ${ }^{1}$ \\ ${ }^{1}$ Department of Animal, Wildlife and Grassland Science \\ ${ }^{2}$ Department of Microbial, Biochemical and Food Biotechnology \\ P.O. Box 339, University of the Free State, Bloemfontein 9300, South Africa
}

(Received 4 January 2014; Accepted 5 June 2014; First published online 23 August 2014)

\begin{abstract}
Copyright resides with the authors in terms of the Creative Commons Attribution 2.5 South African Licence.
See: http://creativecommons.org/licenses/by/2.5/za

Condition of use: The user may copy, distribute, transmit and adapt the work, but must recognise the authors and the South African Journal of Animal Science.
\end{abstract}

\begin{abstract}
This study was conducted to investigate the effect of dietary fatty acids (FA) on the fertility and hatchability of laying hens at the end-of-lay period (69 - 77 weeks of age). Five isoenergetic (12.4 MJ ME/kg $\mathrm{DM})$ and isonitrogenous (170 g CP/kg DM) diets were formulated using different lipid sources (30 g/kg inclusion) to manipulate the dietary FA profile. The control diet was formulated using a $50: 50$ blend of linseed and fish oil, while fish oil was used in the polyunsaturated n-3 treatment. Sunflower oil was used in the polyunsaturated $n-6$ treatment, while in the mono-unsaturated n-9 diet high oleic acid (HO) sunflower oil was used. Lastly, tallow was used as a lipid source in the saturated FA diet. One hundred and twenty five hens ( $n=25 /$ treatment) and 50 cockerels ( $n=10 /$ treatment) of the Hy-Line Silver-Brown genotype were randomly allocated to the five dietary treatments at 20 weeks of age. From 69 weeks of age, hens were inseminated with $0.06 \mathrm{~mL}$ undiluted semen from cockerels within the same dietary treatment. Between 71 and 78 weeks of age (49 days) a total of 588 eggs-per-treatment were collected, individually marked (date and hen number) and incubated in a single-stage still-air incubator. Eggs were candled on D7 and D14 to determine embryonic mortalities and a $24 \mathrm{~h}$ window for hatching was allowed (D21 $+24 \mathrm{~h}$ ). Although the fish oil treatment resulted in the lowest egg weights $(59.3 \mathrm{~g})$ and fertility $(84.6 \%)$, it recorded the highest hatchability $(76 \%)$. In contrast, the sunflower oil treatment recorded the lowest hatchability $(58.2 \%)$ of all treatments, despite its high egg fertility (89.6\%). Results of the study suggest that the dietary fatty acid content, in particular the n-3 and n- 6 levels, need critical consideration in terms of concentration and ratio in the formulation of breeder diets to limit embryonic mortalities during incubation.
\end{abstract}

Keywords: Chicks, embryo, mortality, mono-, polyunsaturated fatty acids

\# Corresponding author: dewittfh@ufs.ac.za

\section{Introduction}

The evaluations of breeding soundness in the poultry industry remains under scrutiny with a continual strive to limit the subjective assessment of the criteria. Within a commercial breeding flock, the system commonly adopted by producers involves the modification of the male to female ratio and/or male age alteration, based on a number of physical attributes of the male. Wilson et al. (1979) illustrated that the general selection of cockerels based on physical characteristics, such as comb size, body size and shank length, were not accurate enough in predicting cockerel fertility. The decline in flock fertility thus requires critical consideration of current breeding practices. Since an increase in body size impact negatively on male fertility (Reddy \& Sajadi, 1990; Barbato, 1999), numerous studies have been done in an attempt to develop a more scientific, detailed and sustainable method for the evaluation of flock fertility (King et al., 2000; Parker \& McDaniel, 2002). The selection of cockerels based on sperm quality traits has also translated into improved hatchability in the broiler breeder flock (Eslick \& McDaniel 1992; Parker \& McDaniel 2002). However, one of the major factors affecting sperm quality remains the diet composition.

Dietary lipid sources affect cockerel sperm composition and functionality differently, mainly due to the specific proportions of sperm fatty acids (Bongalhardo et al., 2009). Omega-6 (n-6) type fatty acids are more prevalent in cockerel sperm (Cerolini et al., 2003) than omega-3 (n-3) type fatty acids, which are predominant in mammalian sperm (Kelso et al., 1997). Studies have shown that dietary fatty acids are deposited proportionately in the sperm (Kelso et al., 1997; Cerolini et al., 2003; 2006). This means that regardless of the type of dietary oil consumed, the proportion of total relative abundance of saturated fatty 
acids (SFA's), mono-unsaturated fatty acids (MUFA's) and polyunsaturated fatty acids (PUFA's) in the sperm do not change, even if the arrangements of fatty acids within a group are altered.

Aside from its influence on cockerels (sperm quality) and hens (egg fatty acid content) fatty acids in general also contribute to chick embryonic development. The main energy source for the developing chick embryo is supplied by fats in the egg yolk. The alteration of the egg yolk fatty acid profile could therefore be detrimental to the growth and development of the embryo during the incubation period (Aydin \& Cook, 2009). However, some studies (Cerolini et al., 2003; 2006) reported that PUFA fatty acids, especially of the n-3 type, may be beneficial in reducing embryonic losses during incubation. The aim of the present study was thus to evaluate effects of different dietary lipid sources on flock fertility and hatchability during the end-of-lay period (weeks 69 - 77 of age).

\section{Materials and Methods}

Seventeen week-old Hy-Line Silver-Brown layer type pullets $(n=125)$ and cockerels $(n=50)$ were purchased from a commercial pullet producer, and all birds were individually housed in cages (1600 $\mathrm{cm}^{3} /$ bird). From 22 weeks of age, all cockerels were trained for semen collection according to the massage technique first described by Burrows and Quinn (1937). At onset of the experimental period (32 weeks of age), the cockerels ( $n=10 /$ treatment) and the hens $(n=25$ hens/treatment) were randomly assigned to one of the following five isoenergetic (12.4 MJ AME/kg DM) and isonitrogenous (170 g CP/ $\mathrm{kg} \mathrm{DM}$ ) dietary treatments: The (i) control diet consisted of a 50:50 blend of linseed- and fish oil, (ii) fish oil diet (n-3 polyunsaturated fatty acids), (iii) sunflower oil diet ( $\mathrm{n}-6$ polyunsaturated fatty acids), (iv) high oleic acid (HO) sunflower oil diet (n-9 mono-unsaturated fatty acids) and ( $v$ ) tallow diet (saturated fatty acids). Birds had free access to water, while the supply of feed was limited to $110 \mathrm{~g} / \mathrm{bird} /$ day - to prevent obesity in the cockerels. A photoperiod schedule of 16 hours light and 8 hours darkness (16L : 8D) was provided from 22 weeks of age. The fatty acid methyl esters (FAME) of the experimental dietary treatments were determined by extracting the fat content thereof (Folch et al., 1957). Individual fatty acids were expressed as the relative percentage of individual fatty acids of the total fatty acids, present in the feed sample. Between the ages of 69 to 77 weeks, flock fertility was evaluated by means of artificially insemination (AI) of hens ( $n=$ 25/treatment), with undiluted semen for the determination of chick hatchability. Semen of the cockerels within a specific treatment group $(n=10 /$ treatment) was collected and individually assessed for ejaculate volume, sperm motility and viability (Siudzinska \& Lukaszewicz, 2008), before being pooled within a treatment and used for artificial insemination of the hens, within the corresponding dietary treatment. An insemination dose of $0.06 \mathrm{~mL}$ undiluted semen was deposited approximately 2 to $4 \mathrm{~cm}$ into the hen's vagina using an automatic pipette, following the venting of the vaginal orifice (Sadanand et al., 2004). To ensure sufficient sperm buildup in the sperm storage glands of the hens, artificial insemination of the hens (onset of week 69 of age) commenced two weeks prior to the collection of eggs for the incubation trial (week 71 of age).

All clean intact eggs (devoid of excreta and eggshell cracks) with weights of 51 to $70 \mathrm{~g}$ were marked with the date of collection and the specific hen number on a daily basis (Mortola \& Al Awam, 2010). Collected eggs were stored $\left(14^{\circ} \mathrm{C} ; 65 \% \mathrm{RH}\right)$ for a maximum period of three days, before setting them in a still-air incubator at $37.5^{\circ} \mathrm{C}$ and $60 \% \mathrm{RH}$. Twelve eggs per treatment per day ( $\mathrm{n}=12 /$ treatment/day) for a period of 49 days were collected for incubation purposes. In total, 588 eggs per treatment $(n=$ $588 /$ treatment) were incubated during the study. The date of egg setting in the incubator was recorded as embryonic day zero (E0). Incubated eggs were candled on embryonic day 7 (E7) to identify infertile eggs and early (D0-7) embryonic mortalities (Aydin \& Cook, 2009). Candling of the remainder of the eggs were repeated on embryonic day 14 (E14), for the detection and removal of mid-mortalities (D7-14). Candling was conducted by quickly removing the eggs from the incubator into a dark, heated $\left(35^{\circ} \mathrm{C}\right)$ enclosure, where a handheld light (250 lumen) was directed at the surface of each egg to identify the development of blood veins and to evaluate the size of the embryo. Eggs on day 7 (E7) that appeared 'clear' under the light were removed and classified as infertile. Subsequently, the break-out analysis of the egg and morphological identification revealed a small white germinal disc, with no blood veins. 'Clear' eggs having an enlarged germinal disc with no blood veins, or no "dark eye" were classified as early deaths, according to the technique described by Wilson (1991).

On embryonic day 18 (E18), eggs were transferred from the incubator into individual permeable net bags, and placed inside a hatcher at $36.5^{\circ} \mathrm{C}$, with a $\mathrm{RH}$ of $65 \%$. The individual placement of eggs inside a permeable net bag would facilitate the weighing of the individual chick after hatching. A "hatching window" of 24 hours was allowed before the hatcher was opened to remove all the chicks and unhatched eggs. Chicks that "completely hatched" from their shells within the allowed time (D21 $+24 \mathrm{~h})$ of incubation, were individually weighed. An egg break-out analysis was performed on all the eggs that had not hatched, in an attempt to categorize the reasons for non-hatching. The three categories used for classifying the "nonhatched" eggs were (i) middle deaths, (ii) late deaths and (iii) pips (fully developed chicks with a broken shell 
that failed to hatch completely). The embryonic development classification system as described by Eslick \& McDaniel (1992), was used with certain minor modifications for the calculation of chick (i) hatchability and (ii) viability. Individual chick weights at hatching were also expressed as a percentage of the initial egg weight, at setting of the eggs for incubation. The effects of dietary fatty acids on fertility and hatchability were statistically analysed (SAS, 2012) according to the least square means procedures (LSM). Eggs collected to partake in the incubation study from the inseminated hens were individually considered and assigned a dummy binary 0 or 1 - depending on the negative or positive state under the following variables, namely: (i) fertility, (ii) early deaths, (iii) mid-deaths, (iv) late deaths and (v) hatchability - as suggested by Harvey (1982) for the statistical analysis of discrete data.

\section{Results and Discussions}

The results regarding the effect of dietary fatty acid profile on fertility and hatchability are set out in Table 1. The recorded egg weight (average $62.5 \mathrm{~g}$ ) of the sunflower oil treatment was the highest $(P<0.001)$ - while that of the fish oil treatment were the lowest (average $59.3 \mathrm{~g})$. The highest $(P<0.05)$ egg fertility $(\%)$ was recorded in the control and the $\mathrm{HO}$ sunflower oil treatments, whereas the fish oil treatment resulted in the lowest fertility (84.6\%). In terms of embryonic mortalities, the sunflower oil treatment resulted in the highest $(P<0.05)$ early $(8.7 \%)$ and mid $(17.0 \%)$ embryonic mortalities. The fish oil treatment recorded the lowest $(2.8 \%$ and $9.3 \%$, respectively) mortalities, for the two respective time periods (D0-7 and D7-14, respectively).

Interestingly, although not statistically different $(P>0.05)$, the fish oil treatment also resulted in the numerical lowest mortalities (6.8\%) during late (D14-21) incubation, as well as "pipping" (2.0\%). The hatchability, expressed as the percentage of both the (i) fertile eggs and (ii) total eggs set, was affected $(P$ $<0.05)$ by the dietary lipid sources. As the fish oil $(n-3)$ treatment resulted in the lowest embryonic mortalities in the different embryonic developmental stages, it subsequently resulted in the highest $(P<0.05)$ recorded hatchability of fertile eggs (75.9\%), together with the highest hatchability of total eggs set (63.8\%).

The lowest $(P<0.05)$ hatchability of fertile $(58.2 \%)$ and total eggs set $(51.1 \%)$ was recorded in the sunflower oil treatment, while intermediate hatchability results were recorded for the other dietary treatments. Table 1 also sets out the chick characteristics in relation to the number of incubated eggs. Although differences $(P<0.001)$ were recorded in chick weights at hatching, no significant differences $(P=0.067)$ in the ratio of chick weight to egg weight at setting of the eggs, were recorded. The trend in hatched live chicken weight between dietary treatments tended to be similar to that observed in the original weights of the incubated eggs. Hence, eggs from the sunflower oil (n-6) treatment resulted in the heaviest chicks at hatching (average $40.1 \mathrm{~g}$ ), while the smallest chicks (average $37.6 \mathrm{~g}$ ) were hatched from eggs of the fish oil $(n-3)$ treatment. Although results regarding the fertility of eggs in the present study can be considered to be slightly lower than in younger flocks (42 - 47 weeks of age) fed maize oil or fish oil diets, as described by Blesbois et al. (1997), the hatchability of eggs was in general low for all dietary treatments, in the present study. Investigations regarding egg fertility in a broiler flock (Fasenko et al., 1992) and that of turkeys (Fairchild et al., 2002) have established a consistent increase of fertility and hatchability up to a peak, before declining as the flock aged. Pedroso et al. (2005) reported an increase from $85.3 \%$ to $96.8 \%$ in fertility as Cobb parent stock birds aged from pullet (25 - 27 weeks of age) to breeders (32 - 37 weeks of age). Kelso et al. (1997) also reported a consistent decline in the fertility of broiler breeder cockerels after peaking at week 39 of age. However, $65.4 \%$ (soya bean oil) and 69.5\% (linseed oil) fertility rates for 72 week old cockerels was recorded by Kelso et al. (1997) - being lower than that of the fish oil (84.6\%) treatment recorded in the present study. The moderate dietary inclusion level (3\%) of fish oil may not have been responsible for the low fertility (84.6\%) recorded in the present study, since Blesbois (1997) reported that cockerels maintained on a $5 \%$ fish oil diet were more $(P<0.0001)$ fertile $(96.0 \%)$ than their counterparts maintained on a $5 \%$ maize oil diet (91.6\%). The fertility of the fish oil (n-3) treatment in the present study, however, showed a strong similarity to the fertility rate (83.7\%) reported by Herstad et al. (2000) in broiler breeders (from 26 to 32 weeks of age), who received a diet with a $3 \%$ supplementary fish oil inclusion.

Marked differences occurring in the fertility rates of the two diets containing n-3 fatty acids (e.g. control and fish oil treatments) in the present study was unexpected and is difficult to explain. Blesbois et al. (1997) and Cerolini et al. (2006) reported that sperm fatty acid methyl esters (FAME) with a higher concentration of long chain fatty acids, such as docosahexanoic acid (DHA) and eicosapentaenoic acid (EPA), are positively associated with sperm motility, which may translate into higher fertility rates. Flock fertility is naturally regarded as the responsibility of the male chicken; thus, attempts have been made to relate sperm motility to the fertility of the flock. The n-3 fatty acids (from fish oil sources in particular) have the potential of contributing positively to the cockerel's reproductive performance at an old age (Blesbois et al., 2004). 
Table 1 Least square means ( \pm SE) of dietary lipid sources on the fertility (\%), mortality (\%) and hatchability (\%) and laying hens at end-of-lay (week $69-77$ of age)

\begin{tabular}{|c|c|c|c|c|c|c|}
\hline & Control & Fish oil & Sunflower oil & HO ${ }^{\#}$ sunflower oil & Tallow & $P$ \\
\hline Egg weight (g) & $61.56^{\mathrm{b}} \pm 0.25$ & $59.25^{\mathrm{C}} \pm 0.21$ & $62.49^{\mathrm{a}} \pm 0.22$ & $61.06^{b} \pm 0.23$ & $60.96^{b} \pm 0.24$ & 0.001 \\
\hline Fertility (\%) & $92.14^{\mathrm{a}} \pm 1.43$ & $84.57^{c} \pm 1.90$ & $89.63^{\mathrm{ab}} \pm 1.64$ & $92.09^{\mathrm{a}} \pm 1.43$ & $86.28^{b c} \pm 1.82$ & 0.008 \\
\hline${ }^{1}$ Mortality $_{\mathrm{D}-7}(\%)$ & $4.78^{\mathrm{abc}} \pm 1.13$ & $2.81^{c} \pm 0.87$ & $8.07^{a} \pm 1.46$ & $6.78^{\mathrm{ab}} \pm 1.33$ & $4.78^{b c} \pm 1.13$ & 0.022 \\
\hline${ }^{2}$ Mortality $_{D 7-14}(\%)$ & $16.29^{a} \pm 1.96$ & $9.27^{\mathrm{b}} \pm 1.54$ & $17.00^{\mathrm{a}} \pm 2.02$ & $13.84^{\mathrm{ab}} \pm 1.83$ & $10.36^{\mathrm{b}} \pm 1.62$ & 0.004 \\
\hline${ }^{3}$ Mortality $_{D 14-21}(\%)$ & $10.39 \pm 1.62$ & $6.81 \pm 1.27$ & $9.80 \pm 1.60$ & $7.63 \pm 1.41$ & $7.56 \pm 1.40$ & 0.197 \\
\hline${ }^{4}$ Mortality $_{\text {Pips }}(\%)$ & $2.25 \pm 0.79$ & $1.97 \pm 0.73$ & $3.17 \pm 0.94$ & $3.67 \pm 1.00$ & $1.96 \pm 0.73$ & 0.499 \\
\hline${ }^{5}$ Hatchability (\%) & $62.32^{\mathrm{bc}} \pm 2.70$ & $75.97^{\mathrm{a}} \pm 2.46$ & $58.21^{c} \pm 2.81$ & $65.50^{b c} \pm 2.63$ & $70.95^{\mathrm{ab}} \pm 2.62$ & 0.006 \\
\hline${ }^{6}$ Hatchability (\%) & $58.43^{\mathrm{ab}} \pm 2.62$ & $63.79^{a} \pm 2.54$ & $51.14^{b} \pm 2.69$ & $61.11^{a} \pm 2.60$ & $61.06^{\mathrm{a}} \pm 2.58$ & 0.005 \\
\hline Chick weight (g) & $39.77^{\mathrm{ab}} \pm 0.25$ & $37.63^{\mathrm{C}} \pm 0.23$ & $40.13^{a} \pm 0.24$ & $39.23^{b} \pm 0.25$ & $39.07^{b} \pm 0.25$ & 0.001 \\
\hline Chick/egg (\%) & $64.98 \pm 0.31$ & $63.58 \pm 0.29$ & $64.48 \pm 0.34$ & $64.90 \pm 0.31$ & $64.43 \pm 0.27$ & 0.071 \\
\hline
\end{tabular}

$\overline{a, b, c}$ Row means with different superscripts differ significantly $(P<0.05)$.

\# High oleic acid.

1 Early embryonic mortalities between days 0-7 of incubation;

2 Mid embryonic mortalities between days 7-14 of incubation;

3 Late embryonic mortalities between days 14-21 of incubation;

${ }^{4}$ Mortalities during hatching (pipping) at $21 \mathrm{~d}+24 \mathrm{~h}$;

${ }^{5}$ Hatchability of fertile eggs (\%);

${ }^{6}$ Hatchability of total eggs set (\%). 
Sperm motility is generally accepted as a good indicator of fertility, due to the high positive correlation between these two parameters (Cerolini et al., 2003). Furthermore, Donoghue (1999) used sperm motility to determine the success rates of sperm-egg binding - a critical stage in the fertilization process. Hence, the group of cockerels whose sperm mobility was classified as good, exhibited a significantly higher fertility rate than the cockerels whose semen were classified as poor. The present study showed a partly similar relationship between sperm motility and fertility for cockerels treated with the experimental diets. The cockerels maintained on the mono-unsaturated (i.e. high oleic acid sunflower oil) and polyunsaturated diets (control, fish oil, sunflower oil) recorded a higher $(P<0.05)$ sperm motility $(55.8 \%-58.7 \%)$ than those on the saturated tallow treatment (51.6\%). Factors other than sperm motility and fatty acid concentration could possibly be responsible for the unexpectedly low fertility recorded in the fish oil treatment.

Contrary to the findings of Alsobayel \& Albadry (2012), egg fertility in the present study was not particularly related to early embryonic deaths. It appears as if treatments with the highest fertility also yielded the highest early (D0-7) embryonic mortalities, except in the control treatment, where high fertility and low early embryonic mortalities were recorded. Following the negative correlation between egg fertility and early embryonic mortalities, Fairchild et al. (2002) reported that factors aimed at improving flock fertility - dietary fatty acids in the present study - could be helpful in reducing early embryonic mortalities. In addition to the occurrence of infertile eggs, early embryonic mortality was also associated with sperm quality (Bramwell \& Howart, 1997). Bramwell \& Howart (1997) specifically associated early embryonic deaths in chickens and increased sperm penetration of the germinal disc, with high sperm concentration. It was, however, difficult to establish the relationship between sperm quality and early embryonic mortality in the present study, as the penetration of sperm into the inner perivitelline layer of the egg yolk was not microscopically determined.

The general low hatchability of set eggs indicates that alternative factors, other than that directly affecting flock fertility must be considered. Attention must therefore be shifted from the effect of sperm quality to adopted procedures of incubation, as well as the effect of dietary lipid sources on hen production performance (particularly egg size) and embryonic survivability. The saturated fatty acid concentration was not increased to an extreme level $(<41 \%)$ in the yolk of any of the treatments in the present study. Aydin and Cook (2009) reported that the uncharacteristically high (57\%) saturated fatty acid concentration in the yolk of hens fed a diet containing $5 \mathrm{~g} / \mathrm{kg}$ conjugated linoleic acid (CLA) resulted in the $0 \%$ hatchability of fertile eggs after seven days of incubation (D0-7). From Table 1, it is evident that the highest occurrence of embryonic mortalities were recorded in the middle (D7-14) followed by the late (D14-21) incubation stages. Apparently, the number of times eggs were removed from the incubator for the purpose of candling and transfer to the permeable net-bags, before placement into the hatcher, resulted in more embryonic mortalities. The frequency of candling and exposure of eggs to environmental conditions outside the incubator may thus have adverse effects on the eventual hatchability - as reflected by the high number of embryonic mortalities that occurred after the $2^{\text {nd }}$ candling (middle and late deaths), in the present study. However, irrespective of the general high number of middle and late mortalities recorded across treatments, it was also noted that embryonic mortalities were significantly higher $(P<0.05)$ in the early $(\mathrm{D} 0-7)$ and middle (D7-14) stages of incubation for the sunflower (n-6) treatment group. This culminated in the lowest egg hatchability for this particular dietary treatment. The average egg weight $(62.5 \mathrm{~g})$ of hens maintained on the sunflower treatment was higher $(P<0.05)$, compared to that of the other treatments (Table 1$)$. Egg size has been implicated in the reduced hatchability rates of incubated eggs (Asuquo \& Okon, 1993). Furthermore, Abiola et al. (2008) reported a $97 \%$ hatchability for medium size eggs $(51-57 \mathrm{~g}$ ), while $83 \%$ was reported for large eggs (57.4 - $69.6 \mathrm{~g})$. The results are complemented by that of Asuquo \& Okon (1993), who reported the best hatchability with medium size eggs (45 - $56 \mathrm{~g}$ ). Egg weights could thus have played a role in the variation in terms of hatchability, especially when the significant difference in egg weight appeared to follow an opposite hatchability trend (Table 1). Mortola \& Al Awam (2010) proposed that the challenges of over-hydration occurring in large eggs may hamper the survival rates of the embryos during the incubation process.

The implication of egg size at set was quite evident in the chick weight at hatching. Chick weight patterns were similar to the differences recorded in egg weight, between the dietary treatments. The present results confirm the reports of various researchers in terms of the positive relationship that exists between egg size and the corresponding weight of the hatching chick (Ipek \& Dikem, 2007; Abiola et al., 2008).

\section{Conclusions}

Results from the present study indicated that dietary lipid sources affected the fertility of the chicken. Inclusion levels of either $1.5 \%$ fish oil $+1.5 \%$ linseed oil or $3 \% \mathrm{HO}$ sunflower could be used in maintaining sperm motility, at the end of the production cycle. Similarly, a high fertility, as well as an acceptable level of chick hatchability could be achieved in older birds ( $\geq 69$ weeks of age) by feeding either n-3 (linseed and fish oil) or n-9 (HO sunflower oil) supplementary lipid sources. Finally, it can be said that the n-3, n-6 and n-9 
type dietary fatty acids needs critical consideration, both in terms of dietary concentration and ratio in the formulation of breeder diets in order to limit embryonic mortalities during incubation.

\section{References}

Abiola, S.S., Meshioye, O.O., Oyerinde, B.O. \& Bamgbose, M.A., 2008. Effect of egg size on hatchability of broiler chicks. Arch. Zoo. Technol. 57, 217, 83-86.

Alsobayel, A.A. \& Albadry, M.A., 2012. Effect of age and sex ratio on fertility and hatchability of Baladi and Leghorn laying hens. J. Anim. Plant Sci. 22 (1), 15-19.

Asuquo, B.O. \& Okon, B., 1993. Effects of age in lay and egg size on fertility and hatchability of chicken eggs. Nig. J. Anim. Prod. 20, 122-124.

Aydin, R. \& Cook, M.E., 2009. The effect of dietary conjugated linoleic acid alone or in combination with linoleic acid and oleic acid on fatty acid composition of egg yolk, embryo mortality and chick yolk sac content retention in chickens. Anim. Feed Sci. Technol. 149, 125-134.

Barbato, G.F., 1999. Genetic relationships between selection for growth and reproductive effectiveness. Poult. Sci. 78, 444-450.

Blesbois, E., Lessire, M., Grasseau, I., Hallouis, J.M. \& Hermier, D., 1997. Effect of dietary fat on the fatty acid composition and fertilizing ability of fowl semen. Biol. Reprod. 56, 1216-1220.

Blesbois, E., Douard, V., Germain, M., Boniface, P. \& Pellet, F., 2004. Effects of n-3 polyunsaturated dietary supplementation on the reproductive capacity of male turkeys. Theriogenology 61, 537-549.

Bongalhardo, D.C., Leeson, S. \& Buhr, M.M., 2009. Dietary lipids differentially affect membranes from different areas of rooster sperm. Poult. Sci. 88, 1060-1069.

Bramwell, R.K. \& Howarth, B., 1997. Effect of low or high sperm penetration values at the germinal disc on early embryonic mortality in chicken eggs. Poult. Sci. 76, 97. (Abstr.)

Burrows, W.H. \& Quinn, J.P., 1937. Artificial insemination of chicken and turkeys. Poult. Sci. 14, 251-254.

Cerolini, S., Pizzi, F., Gliozzi, T., Maldjian, A., Zaniboni, L. \& Parodi, L., 2003. Lipid manipulation of chicken semen by dietary means and its relation to fertility: a review. Wrld Poult. Sci. J. 59 (1), 65-75.

Cerolini, S., Zaniboni, L., Maldjian, A. \& Gliozzi, T., 2006. Effect of docosahexaenoic acid and a-tocopherol enrichment in chicken sperm on semen quality, sperm lipid composition and susceptibility to peroxidation. Theriogenology $66,877-886$.

Donoghue, A.M., 1999. Prospective approaches to avoid flock fertility problems: Predictive assessment of sperm function traits in poultry. Poult. Sci. 78, 437-443.

Eslick, M.L. \& McDaniel, G.R., 1992. Interrelationship between fertility and hatchability of eggs from broiler breeder hens. J. Appl. Poult. Res. 1, 156-159.

Fairchild, B.D., Christensen, V.L., Grimes, J.L., Wineland, M.J. \& Bagley, L.G., 2002. Hen age relationship with embryonic mortality and fertility in commercial turkeys. J. Appl. Poult. Res. 11, 260-265.

Fasenko, G.M., Hardin, R.T. \& Robinson, F.E., 1992. Relationship of hen age and egg sequence position with fertility, hatchability, viability, and pre-incubation embryonic development in broiler breeders. Poult. Sci. 71, 1374-1382.

Folch, J., Lees, M. \& Sloane-Stanely, G.H., 1957. A simple method for the isolation and purification of total lipids from animal tissues. J. Biol. Chem. 265, 497-507.

Harvey, W.R., 1982. Least-squares analysis of discrete data. J. Anim. Sci. 54, 1067-1071.

Herstad, O., Oerland, M., Haug, A., Skrede, A., Skinlo Thomassen, M. \& Egaas, E., 2000. Reproductive performance of broiler breeder hens fed n-3 fatty acid-rich fish oil. Acta Agric. Scand. 50, 121-128.

Ipek, A. \& Dikem, B.Y., 2007. The relationship between growth traits and egg weight in pheasants ( $P$. colchicus). J. Biol. Environ. Sci. 1, 117-120.

Kelso, K.A., Cerolini, S., Speake, B.K., Cavalchini, L.G. \& Noble, R.C., 1997. Effects of dietary supplementation with a-linolenic acid on the phospholipid fatty acid composition and quality of spermatozoa in cockerel from 24 to 72 weeks of age. J. Reprod. Fertil. 110, 53-59.

King, L.M., Holsberger, D.R. \& Donoghue, A.M., 2000. Correlation of CASA velocity and linearity parameters with sperm mobility phenotype in turkeys. J. Androl. 21, 65-71.

Mortola, J.P. \& Al Awam, K., 2010. Growth of the chicken embryo: Implications of egg size. Comp. Biochem. Physiol. 156, 373-379.

Parker, H.M. \& McDaniel, C.D., 2002. Selection of young broiler breeders for semen quality improves hatchability in an industrial field trial. J. Appl. Poult. Res. 11, 250-259.

Pedroso, A.A., Andrade, M.A., Cafe, M.B., Leandro, N.S.M., Menten, J.F.M. \& Stringhini, J.H., 2005. Fertility and hatchability of eggs laid in the pullet-to-breeder transition period and in the initial production period. Anim. Reprod. Sci. 90, 355-364. 
Reddy, R.P.K. \& Sajadi, I., 1990. Selection for growth and semen traits in the poultry industry: what can we expect in the future. In: Proceedings of the Control of Fertility in Domestic Birds, Ed: INRA, Paris. pp. 47-59.

Sadanand, D., Sontakke, G.U., Vedantam, S., Sanjiva, D. \& Kholkute, S.S., 2004. Semen characteristics, cryopreservation and successful artificial insemination in the Blue rock pigeon (Columba livia). Theriogenology 62, 139-153.

SAS, 2012. Statistical Analysis Systems user's guide $\left(\mathrm{SAS}^{\circledR}\right)$. Version 6.12. SAS Institute Inc. Cary, NC, USA.

Siudzinska, A. \& Lukaszewicz, E., 2008. Effects of semen extenders and storage time on sperm morphology of four chicken breeds. J. Appl. Poult. Res. 17, 101-108.

Wilson, H.R., Piesco, N.P., Miller, E.R. \& Nesbeth, W.G., 1979. Prediction of the fertility potential of broiler breeder males. Wrld Poult. Sci. J. 35, 95-118.

Wilson, H.R., 1991. Hatching egg breakout methods are explained. Poult. Digest. 50 (9), 20-25. 